\title{
Migration of etonogestrel contraceptive implants: Implications for difficult removals services need in southern Africa
}

\author{
G A Petro, ${ }^{1}$ MB ChB, FCOG (SA); T Spence, ${ }^{1}$ MB ChB, FCOG (SA); J-P du Plessis, ${ }^{2}$ MB ChB, FC Orth (SA); A M Gertz, ${ }^{3,4}$ MD, MPH; \\ C Morroni, ${ }^{4,5,6,7} \mathrm{MB}$ ChB, MPH, DFSRH, MPhil, PhD \\ ${ }^{1}$ Department of Obstetrics and Gynaecology, New Somerset Hospital and Faculty of Health Sciences, University of Cape Town, South Africa \\ ${ }^{2}$ Department of Orthopaedic Surgery, New Somerset Hospital and Faculty of Health Sciences, University of Cape Town, South Africa \\ ${ }^{3}$ Botswana Harvard Partnership, Gaborone, Botswana \\ ${ }^{4}$ Botswana UPenn Partnership, Gaborone, Botswana \\ ${ }^{5}$ Department of International Public Health, Liverpool School of Tropical Medicine, Liverpool, UK \\ ${ }^{6}$ Women's Health Research Unit, School of Public Health and Family Medicine, Faculty of Health Sciences, University of Cape Town, South Africa \\ ${ }^{7}$ Wits Reproductive Health and HIV Institute, Faculty of Health Sciences, University of the Witwatersrand, Johannesburg, South Africa
}

Corresponding author: G Petro (gregory.petro@westerncape.gov.za)

\begin{abstract}
The first difficult contraceptive implant removals clinic in sub-Saharan Africa was started 2 years ago at New Somerset Hospital in Cape Town, South Africa, and has seen two cases of implant migration. We report these cases here. The first was a case of fascial migration and the second one of migration via the cephalic vein, both to a site just anterior to the glenohumeral joint. Both implants were removed without complications. Even with correct insertion technique, migrations can occur. Healthcare providers need to know how to manage difficult removals, and how to access and refer to difficult removals services when necessary. These services must therefore be available in all settings where implants are offered, to ensure access to rights-based family planning services for all women in southern Africa.
\end{abstract}

S Afr Med J 2019;109(8):559-561. DOI:10.7196/SAMJ.2019.v109i8.14061

In 2014, the 3-year etonogestrel (ENG) subdermal contraceptive implant (Implanon NXT) became available in South Africa (SA). Over 80000 implants have been inserted to date in Western Cape Province, and over 800000 nationally. ${ }^{[1]}$ High-income country data indicate that the estimated non-palpable (and therefore difficult to remove) implant rate is $1.5-3 \%{ }^{[2]}$ To meet the need for difficult removals, a specialist clinic was established 2 years ago at New Somerset Hospital in Cape Town and has since seen $>300$ patients. ${ }^{[3]}$ Two patients with migrated implants have been referred to the clinic. We describe these two cases of implant migration from southern Africa.

\section{Case reports}

Case 1. Contraceptive implant cephalic vein migration to a site just anterior to the glenohumeral joint

A 32-year-old woman presented to her local community clinic for removal of an expired ENG implant and was referred to our clinic because the implant was impalpable. The implant had been inserted immediately postpartum into the left upper arm at a regional hospital 3 years previously. She reported no adverse effects, and that the implant had never been palpable. She reported no pain, swelling, varicosities or shortness of breath. The findings on physical examination were normal except for a body mass index of $39 \mathrm{~kg} / \mathrm{m}^{2}$. A clear skin marking showed the original insertion site, incorrectly situated on the far lateral aspect of the upper arm. The implant was not palpable and could not be located via ultrasound, so a radiograph was taken.

The radiograph revealed a linear foreign body just above the glenohumeral joint in the area of the deltopectoral groove (Fig. 1). The image was compatible with a radio-opaque ENG implant. Surgical removal of the implant under general anaesthesia was performed on the same day by two of the authors. The patient was

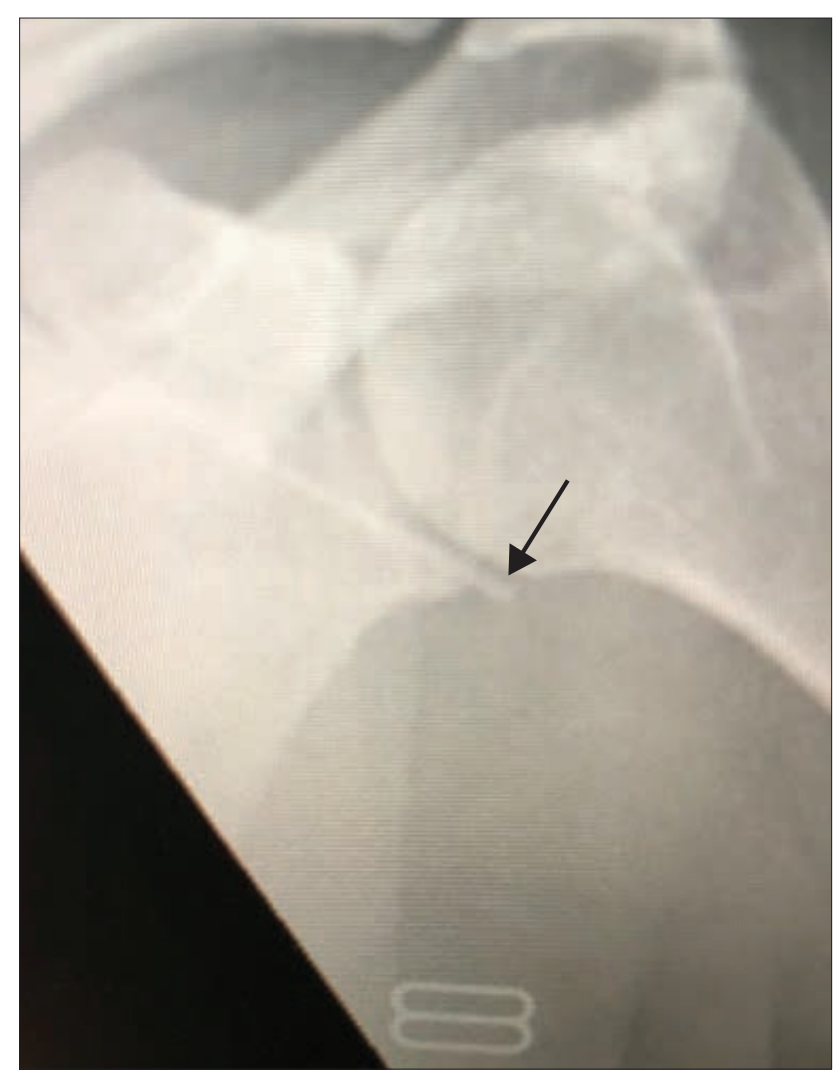

Fig. 1. Case 1. Radiograph of left upper arm demonstrating implant location above the glenohumeral joint, in the deltopectoral groove (arrow indicates tip of implant). 
cleaned and draped in the beach-chair position, and given $2 \mathrm{~g}$ of intravenous cefazolin preoperatively. An X-ray unit with a C-arm was used intraoperatively to locate the implant (lying anterior to the left shoulder) and guide incision (Fig. 2). A $6 \mathrm{~cm}$ incision was made over the deltopectoral groove, directly over the implant; the implant was located with blunt dissection and was found superficially in the deltopectoral groove. Careful dissection revealed that it was within the cephalic vein. The vein, which was sclerosed around the implant, was mobilised and ligated proximal and distal to the implant. The implant was then removed (Fig. 3). The minimal intraoperative bleeding was controlled with electrocautery. The wound was washed and closed with Vicryl 2/0 and subcutaneous Monocryl 3/0 sutures, and dressed in sterile fashion. Postoperatively, the patient was mobilised without restriction. We suspect lateral and deep (not subdermal) insertion into the cephalic vein as the mechanism of migration.

\section{Case 2. Contraceptive implant migration along tissue plane, probably due to weight loss}

A 28-year-old woman was referred to the difficult removals clinic from a community clinic after failed removal of a palpable but



Fig. 2. Case 1. Intraoperative radiograph showing an etonogestrel implant lying anterior to the left shoulder (arrow indicates tip of implant).

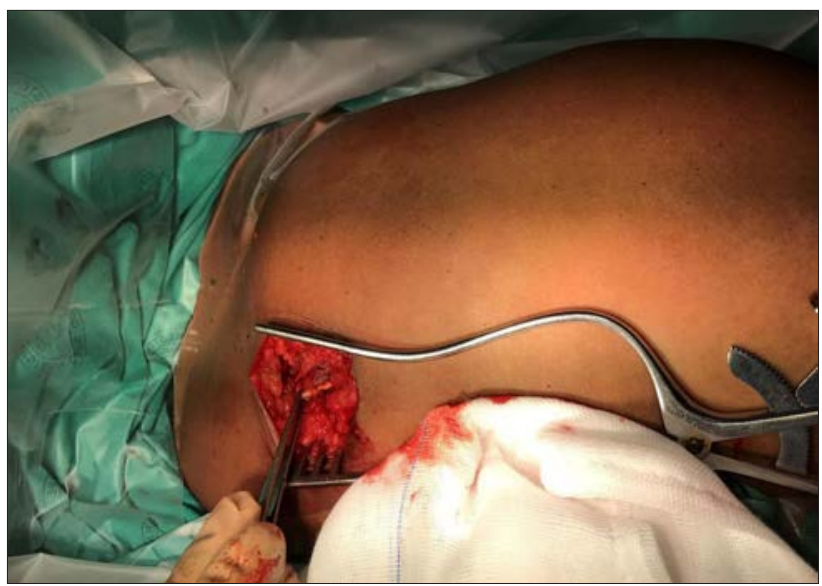

Fig. 3. A $6 \mathrm{~cm}$ incision through which an etonogestrel implant was found and removed from the deltopectoral groove within the cephalic vein.

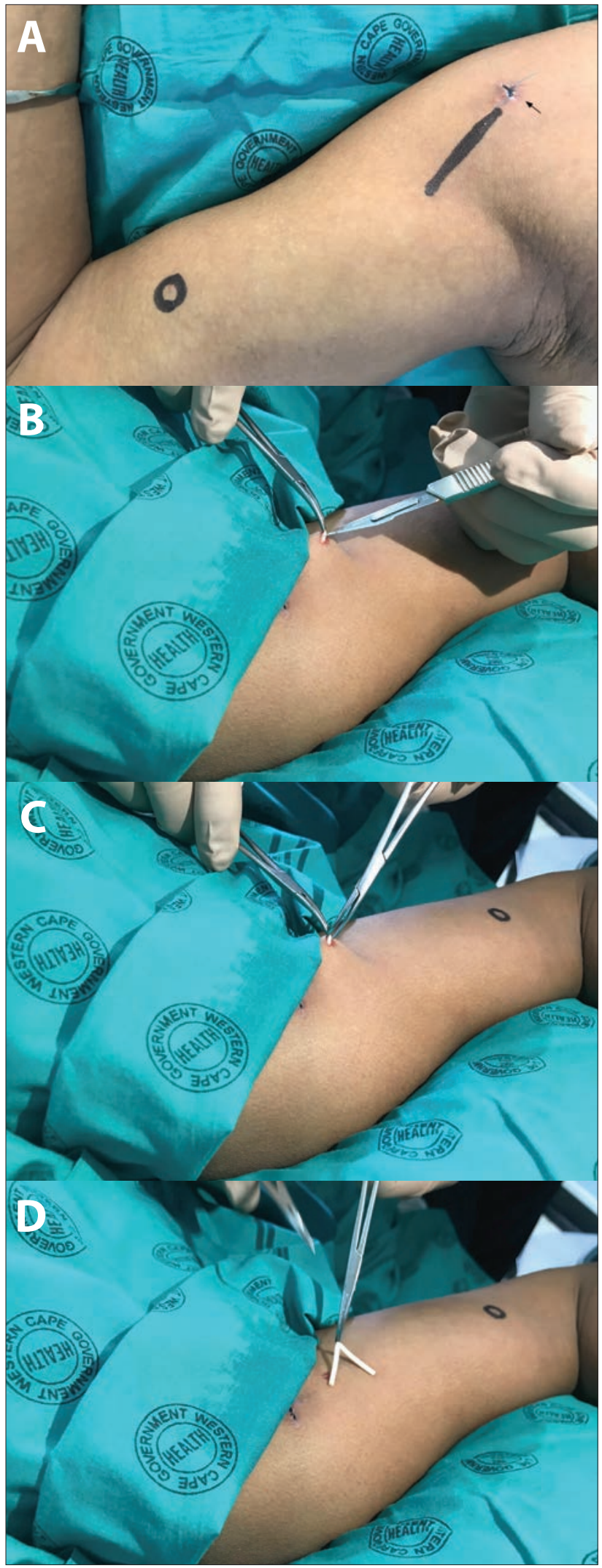

Fig. 4. Case 2. Location and removal of an etonogestrel implant that superficially migrated to the anterior glenohumeral joint. In panel A, the small arrow points to the healing incision through which a general practitioner had attempted removal, the circle marks the original insertion site, and the line indicates location of the implant identified via ultrasound at the referral clinic. $B-D$ show removal of the implant by grasping its midpoint with modified vasectomy clamps and pulling it up through a small incision. 
migrated ENG implant. The implant had been inserted 6 months previously at a community clinic. The patient wanted it removed because of the notable migration from the place of insertion. She reported that it had moved to her right shoulder region gradually, over time. On examination, there was a clear skin marking showing the original insertion site (Fig. $4 \mathrm{~A}$, area marked with circle on skin), which was situated over the short head of the biceps muscle on the right upper arm. The SA National Department of Health's 2014 training recommendation was for ENG implant insertion over the head of the biceps muscle, in the non-dominant arm (South African Training of Trainers Session on Insertions and Removals of Contraceptive Implants, February 2014, Cape Town, SA - unpublished; and as described in Guillebaud ${ }^{[4]}$ ). The implant was easily palpable over the glenohumeral joint, and removal had therefore been attempted in the community clinic by the general practitioner, without imaging. However, the attempt had failed, and the patient was referred. At the difficult removals clinic, the location was confirmed via ultrasound and marked with a line (Fig. 4 A, linear marking). A local anaesthetic, Xylotox $1.8 \mathrm{ml}$, was given over the middle of the implant. A small skin incision was made with a No. 11 blade. The implant was grasped with a modified vasectomy clamp at the midpoint of the implant, and elevated to the surface of the skin (Fig. 4, B and C). The capsule was incised and the implant was easily removed (Fig. 4 D). No skin sutures were required, and the incision was closed with SteriStrips and an Opsite Transparent Adhesive Film Dressing. Although the insertion site and depth were correct according to SA guidance at the time and the implant had been palpable ever since insertion, the patient had lost a significant amount of weight ( $8.5 \mathrm{~kg}$ in 6 months). We hypothesise that weight loss had propagated migration of the implant along the tissue plane of the biceps muscle.

\section{Comment}

Poor insertion technique, usually too deep or at an incorrect site, ${ }^{[3,5]}$ often results in implants being difficult to remove, as well as in their migration ${ }^{[6]}$ However, even with correct insertion, implant migration is still possible, and substantially migrated implants often require specialist services for safe and effective removal. Services for difficult removals need to be established in all settings where contraceptive implants are offered, including in low- and middle-income countries, where in some settings removal training and services have lagged behind the tremendous upscaling of insertion services in recent years. ${ }^{[1,7,8]}$ Community healthcare providers need to know how to approach difficult removals and where and when to refer if they are unable to complete the removals themselves. ${ }^{[3]}$ Without availability of and easy client access to safe and effective implant removal services, quality, rights-based family planning programmes are compromised. Guaranteeing easy access to quality removal services, including for difficult and migrated implants, is essential in order for women to truly choose in a fully informed way among the full range of reversible modern contraceptive methods.

\section{Declaration. None.}

Acknowledgements. This project was made possible by the dedicated nurses and clerks in the Shipley Gynaecology Outpatient Clinic at New Somerset Hospital.

Author contributions. GAP and TS were involved in direct patient care in these cases. J-PdP provided technical expertise and consultation. AMG and $\mathrm{CM}$ provided consultation and writing and editorial assistance. All authors were involved in writing and/or editing and approved the final manuscript.

Funding. AMG's time working on this was supported in part by the Aya Bora Global Health Fellowship Program and the Fogarty International Center of the National Institutes of Health under Award No. D43 TW010543

Conflicts of interest. None.

1. Pleaner M, Morroni C, Smit J, et al. Lessons learnt from the introduction of the contraceptive implant in South Africa. S Afr Med J 2017;107(11):933-938. https://doi.org/10.7196/SAMJ.2017.v107i11.12805 2. Croxatto HB, Urbancsek J, Massai R, Bennink HC, van Beek A. A multicentre efficacy and safety study of the single contraceptive implant Implanon'. Hum Reprod 1999;14(4):976-981. https://doi.
s. org/10.1093/humrep/14.4.97

3. Petro GA. Non-palpable and difficult contraceptive implant removals: The New Somerset Hospital referral-clinic experience. S Afr J Obstet Gynaecol 2017;23(3):101-104. https://doi.org/10.7196/ SAJOG.1229

4. Guillebaud J. Contraception Today. CRC Press, 2011. https://doi.org/10.1201/b15065

5. Chevreau J, Krief D, Abou Arab O, et al. Factors associated with removal difficulties of etonogestrelcontaining contraceptive implants (Nexplanon ${ }^{\circ}$ ). Eur J Obstet Gynecol Reprod Biol 2018;224:81-84 https://doi.org/10.1016/.j.jogrb.2018.03.019

6. Kang S, Niak A, Gada N, Brinker A, Jones SC. Etonogestrel implant migration to the vasculature, chest wall, and distant body sites: Cases from a pharmacovigilance database. Contraception 2017;96(6):439445. https://doi.org/10.1016/j.contraception.2017.08.009

7. Duvall S, Thurston S, Weinberger M, Nuccio O, Fuchs-Montgomery N. Scaling up delivery of contraceptive implants in sub-Saharan Africa: Operational experiences of Marie Stopes International. Glob Health Sci Pract 2014;2(1):72-92. https://doi.org/10.9745/GHSP-D-13-00116

8. Jacobstein R. Liftoff: The blossoming of contraceptive implant use in Africa. Glob Health Sci Pract 2018;6(1):17-39. https://doi.org/10.9745/GHSP-D-17-00396 